\title{
Pelletization Techniques. The Pharma Review
}

Harsha V Sonaye ${ }^{11}$, Mohmad Rafik Y. Shaikh ${ }^{2}$, Rubina Shaikh ${ }^{3}$

*Pharmaceutics , Taywade College of Pharmacy, Nagpur, Maharashtra, India

${ }^{2}$ K.E.M.Hospital Research Centre, Pune, Maharashtra, India

${ }^{3}$ Pharmaceutics, Taywade College of Pharmacy, Nagpur, Maharashtra, India

\section{ABSTRACT}

Pellets are spherical or nearly spherical, free-flowing granules with a narrow size distribution, typically varying between 500 and $1500 \mu \mathrm{m}$ for pharmaceutical applications. They are generally produced via a pelletization process whereby a powder blend consisting of an API and excipients particles is agglomerated into spherical granules. This review article deals with various aspects of the extrusion-spheronization technique. Pelletization is a technique to convert drugs or excipients to small free flowing, spherical or semi spherical units, which are produced by agglomerating fine powdered drugs/ excipients with a binder solution. Pellets range in size, typically, between $0.5-2 \mathrm{~mm}$. In relation to pharmaceuticals, pellets offer high degree of flexibility in design and development of oral dosage form. Pelletization technique help in the formation of spherical beads or pellets having a diameter $0.5-1.5 \mathrm{~mm}$ which can be eventually coated for preparation of modified release dosage form. The manufacturing techniques include Drug layering, Extrusion-Spheronization, Cryopelletization, Compression, Balling, Hot-Melt Extrusion Technology, Freeze pelletization, Spray-drying \& Spray-congealing. Factors affecting pelletization technique and advantages, disadvantages of pellets are discussed.

Keywords : Pellets, Pelletization Technique

\section{INTRODUCTION}

\subsection{Pellets}

Small free flowing spherical unit ranging in size, prepared by agglomeration of fine powders called pellets.

- Their size and shape allow their administration as injection and also for oral drug delivery.

- Pellets range in size typical between 0.5$1.5 \mathrm{~mm}$, though other sizes could be prepared. ${ }^{[1]}$

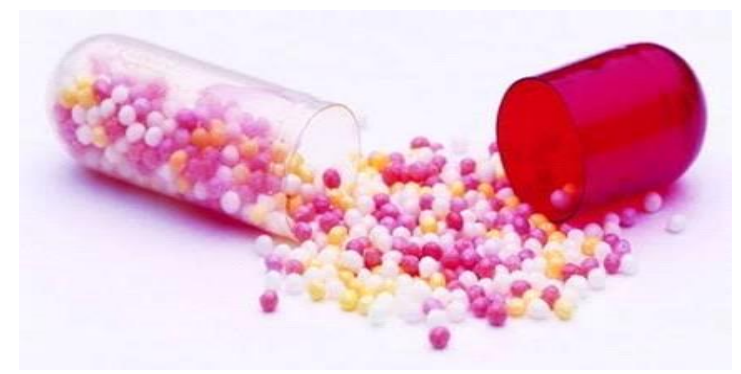

Figure 1. Pharmaceutical Pellets

Pellets as a drug delivery system offer not only therapeutic advantages, such as less irritation of the gastro-intestinal tract and a lowered risk of side effects due to dose dumping but also technological advantages, for example, better flow propertiess, less friable dosage form, narrow particale size distribution, ease of coating and uniform packing. ${ }^{[2]}$. Pellets can be 
prepared by a special technique called Pelletization. This technique is referred to an agglomeration process that convert fine powder or granules of bulk drug or excipient in to small , free flowing, spherical or semi spherical pellets. This technique is needed to produce pellets of uniform size with high drug loading capacity and also prevent segregation and dust ${ }^{[3]}$

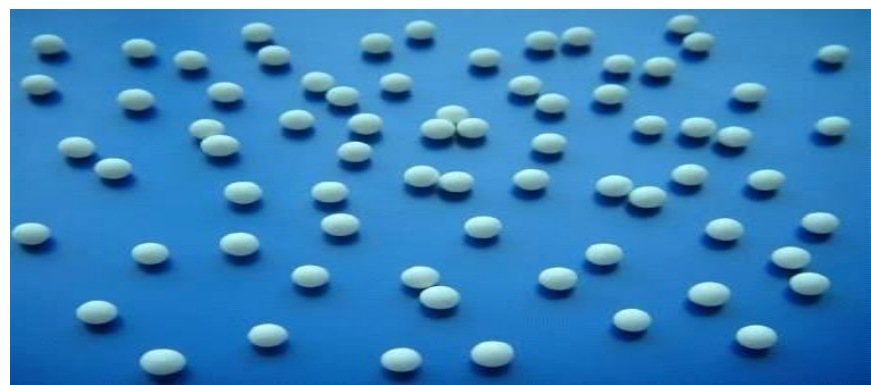

Figure 2. Dosage forms-pellets

\subsection{Characterization of pellets}

- Characterization studies for prepared pellets.

- Optimizing the best formula for each drug, based on in vitro drug release studies.

- Formulating the optimized formula into dosage form

- Characterization studies for final dosage form. ${ }^{[4]}$

\subsection{Why pellets}

- Excellent stability.

- Dust free round pellets.

- Good flow behavior.

- Easy to dose.

- Compact structure.

- Very low hygroscopicity.

- High bulk density. ${ }^{[1]}$

\section{Pelletization}

Pelletization is often referred to as a size-enlargement process that involves the manufacture of agglomerates with a relatively narrow size rang, usually with mean size 0.5 to $2 \mathrm{~mm}$ named pellets.
Pellets are small particles typically created by compressing an original material. ${ }^{[5]}$.Pelletization is a novel drug delivery system; a technique which converts fine powder particles into pellets. These oral multiparticulate drug-delivery systems offer biopharmaceutical advantages with respect to predictable and even distribution and transportation in the gastrointestinal tract. It has many advantages compared to single-unit dosage forms for ease of filling, better flow properties of spherical pellets, sustained, controlled or site-specific drug delivery, even distribution in the GI tract, less GI irritation, good bioavailability, ease of coating and uniform packing. Pelletization is easier and simple technique compared to granulation in order to develop a sitespecific drug delivery system. ${ }^{[6]}$

Multiparticulate dosage forms are gaining much favour over single unit dosage forms because of their potential benefits like predictable gastric emptying, no risk of dose dumping, flexible release patterns, and increased bioavailability with less intra-subject variability ${ }^{[2]}$

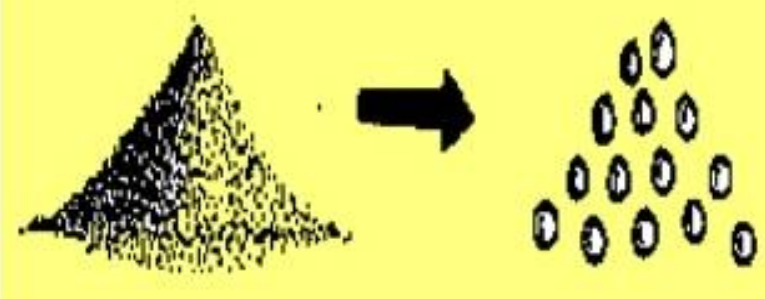

Figure 3. Pellets Formation

In recent years, there has been a growing interest in the field of pelletization to produce spherical pellets which can be changed into several dosages forms like tablet and capsule or can be administered as such. Pelletization involves size enlargement process and if the final agglomerates are spherical in shape in the size range of $0.5-2.0 \mathrm{~mm}$, they are called pellets. ${ }^{[7]}$ 
Pelletization is a novel drug delivery system; a technique which converts fine powder particles into pellets. These oral multiparticulate drug-delivery systems offer biopharmaceutical advantages with respect to predictable and even distribution and transportation in the gastro-intestinal tract. Pelletization can be referred as the conversion of fine powder, granules of drug and excipient by the means of agglomeration to small, free flowing spherical subunit referred as pellets. Which are smaller in size $(0.5-1.5 \mathrm{~mm})$ and intended mostly for oral administration. ${ }^{[8]}$

\section{Historical development}

The term pellet has been used by a number of industries to describe a variety of agglomerates produce from diverse raw materials, using different pieces of manufacturing equipment. These agglomerates include fertilizers, animal feeds, iron ores, and pharmaceutical dosage units and thus do not only differ in composition but also different sizes and shapes. as a meant pellets meant different things for different industries. ${ }^{[9]}$. In industries utilized pelletization processes since the turn of the 20th century in order to manufacture particles with defined sizes and shapes, it was only in the early 1950 's, in response to a desire to sustain the release of drugs over an extended period of time, that the pharmaceutical industry developed a keen interest in the technology. In 1949, pharmaceutical scientists at Smith Kline \& French (SKF) realised the potential of candy seeds in developing sustained-release preparations and began the development of tiny drug pellets that could be loaded into capsules.

Also, pelletization is used in various industries, such as agriculture (fertilizers and herbicides), mineral processing (iron ore pelletization), food and detergent industry. ${ }^{[10]}$

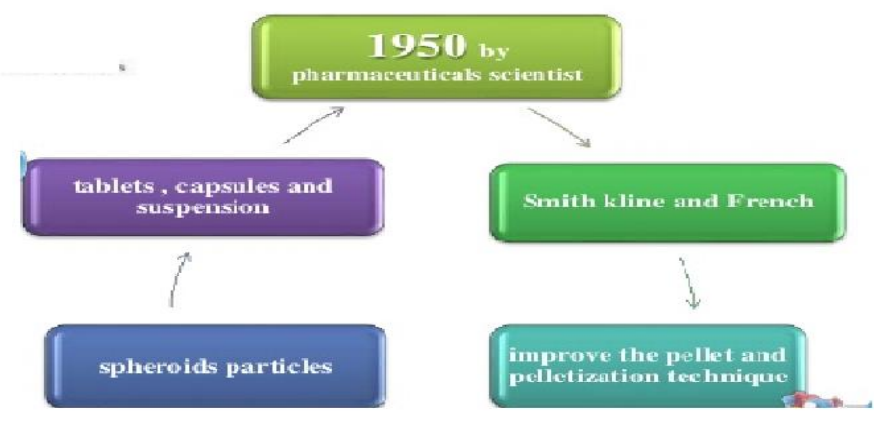

Figure 4. History of pelletization

\section{Advantages}

- They can be divided in to desired dosage strength without process or formulation changes.

- Improve appearance of product.

- Pellets are of small size and have good flow ability compare to powder form.

- Ease of handling, such as filling into capsules

- Incorporation of incompatible ingredients in a single dosage form

- Different release profiles at different sites in the Gastrointestinal tract

- Protection against degradation of active ingredients by oxidation or moisture by providing film coating

- High degree of patient acceptance when filled in capsules due to their elegance as compared to tablets

- Ideal shape for application of film coatings due to low surface to volume ratio

- High drug loading capacity without producing large particles

- Pellets are less susceptible to dose dumping effect and decrease the side effect

- Due to their small size reduction in gastrointestinal irritation compare to tablet

- Pellets reduce variation in gastric emptying rate and intestinal transit time thus reduce inter and intra patient variability

- They can be formulated as sustained, controlled, or site specific delivery of the drug from coated 
pellets.

- Density increase can be achieved as bulk density of powder is increased by spheronization process. This can improve the process and packaging.

\section{Disadvantages}

- Pellets filling involve capsule filling which can increase the costs

- Tableting of pellets destroy film coating on the pellets.

- The size of the pellets may vary formulation to formulation but usually is in range of $0.05 \mathrm{~mm}$ and $2 \mathrm{~mm} \cdot{ }^{[11]}$

\section{Requirements of pellets}

- Pellets should be of spherical shape and the surface should be smooth so that desired uniform film coating can be done.

- Particle size of pellets should be in range 0.5-1.5 $\mathrm{mm}$.

- The quantity of excipients should be maximum so as to maintain the particle size.

\section{Reason for pelletization}

- The pharmaceutical industry has developed a great interest in Pelletization due to a variety of reasons:

- Prevention of segregation of co-agglomerated components, resulting in an improvement of the uniformity of the content.

- Prevention of dust formation, resulting in an improvement of the process safety, as fine powders can cause dust explosions and the respiration of fines can cause health problems.

- Increasing bulk density and decreasing bulk volume.

- The defined shape and weight improves the appearance of the product.
- Improvement of the handling properties, due to the free flowing properties

- Improvement of the hardness and friability of pellets.

Controlled release application of pellets due to the ideal low surface area to volume ratio that provides an ideal shape for the application of film coatings.

\section{Pellets formation and growth}

In order to select and optimize any pelletization/granulation process, it is important to understand the fundamental mechanisms of granule formation and growth. However, based on the experiments on the mechanism of pellet formation and growth, the following steps were proposed: nucleation, coalescence, layering and abrasion transfer. Nucleation is followed by a transition phase, and the growth mechanisms affecting the transition region are coalescence and layering.

Coalescence is defined as the formation of large-sized particles by random collision of well formed nuclei, and the mechanism requires slight excess moisture on the nuclear surface. Although the number of nuclei is progressively reduced, the total mass of the system remains unchanged during this step. The particles, however, undergo a continuous change in size as long as the conditions that lead to the transfer of material exist. ${ }^{[12]}$

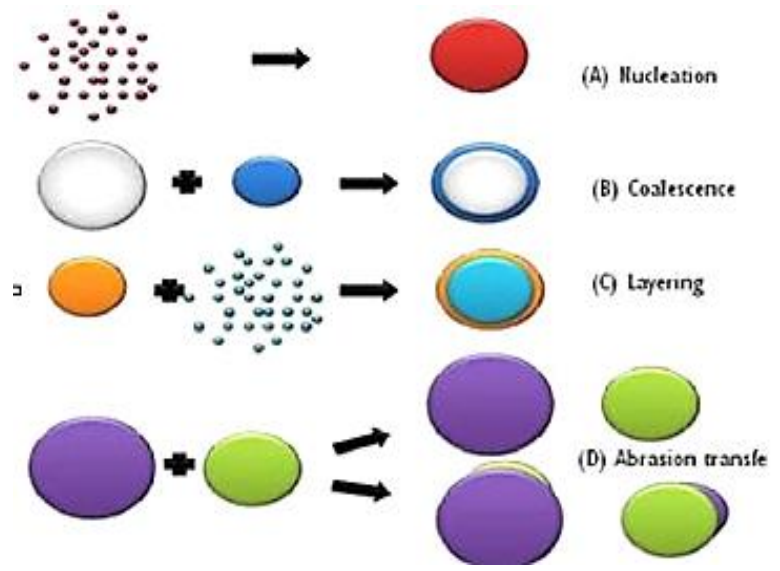

Figure 5. Pellet growth mechanism. 


\section{A] Nucleation B] Coaescence C]Layering} Abrasion transfer.

\section{Pelletization V/S Granulation:}

- Pelletization produces spheroids with high loading API than granules as it require excipients.

- Pelletization is simple, requires less equipments and fast processing than granulation.

- Coating of pellets is easier than coating granules due to their spherical shape and low surface area to volume ratio.

- Pelletization gives better results in formulation of sustained release and controlled release delivery system than granulation.

- Pelletization gives high through put with low wastage unlike in granulation.

- Pellets are easier to produce of uniform shape, size, good flow properties, reproducibility in packing, high strength, low friability and smooth surface with high drug loading capacity which is not possible with granulation. ${ }^{[6]}$

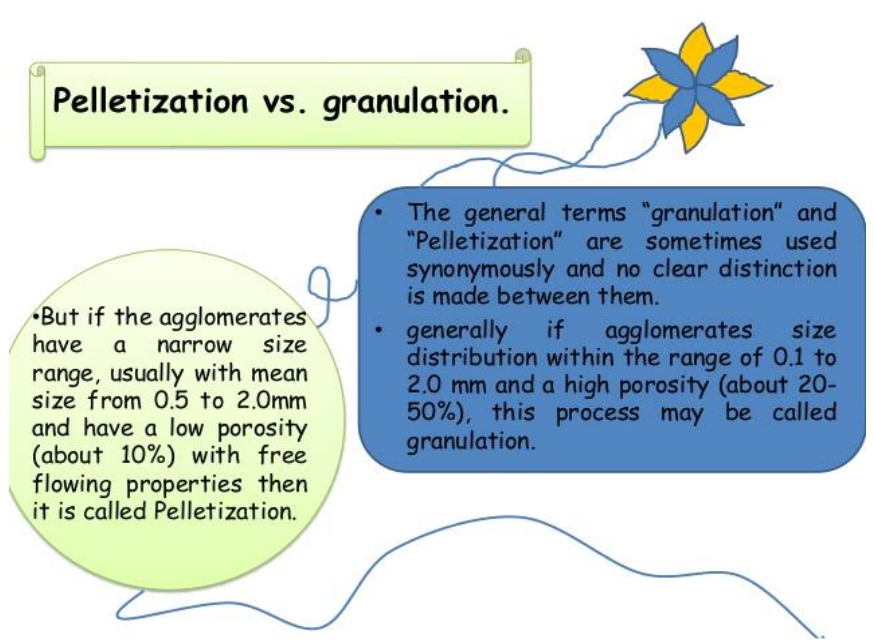

Figure 6. Pelletization vs granulation
10. Method of pelletization

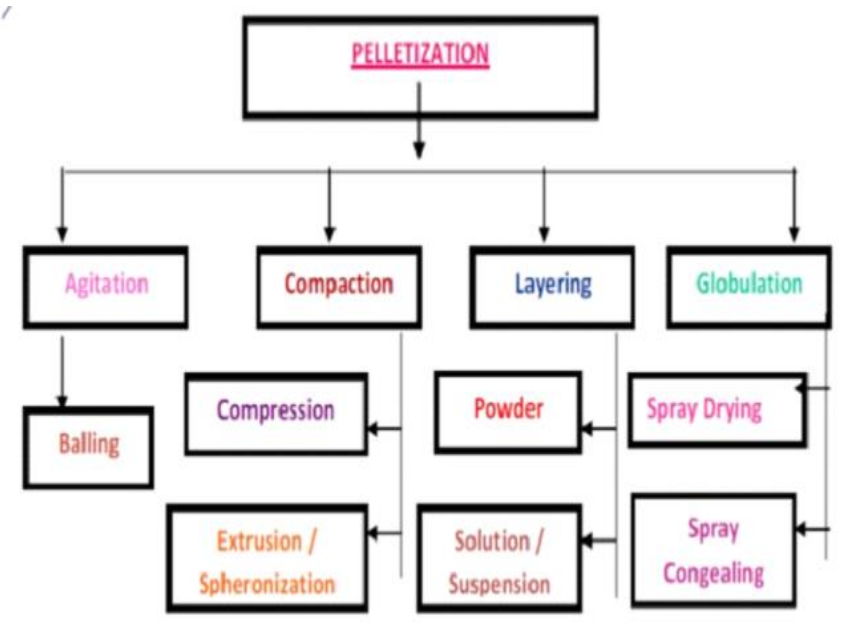

Figure 7. Method of pelletization

\subsection{Agitation}

In finely divided particles are converted to spherical particles, upon the addition of appropriate quantity of liquid, by a contineus rolling or thombing motion.

The liquid may be added to or during the agitation stage. Pans, discs, drums, or mixtures may be used to produce pellets by the balling process. ${ }^{[7]}$

\subsubsection{Balling}

Also known as spherical agglomeration is a pelletisation technique in which finely divided particle or powder upon addition of an appropriate quantity of liquid are converted to spherical particle by continuous rolling or tumbling action.

They can be done either by adding an appropriate amount of liquid into powder called liquid induced agglomeration or subjecting it to high temperature called melt induced agglomeration. Round curvature pans, horizontal drum mixer, and rotatory fluid bed granulator can be used for the production of spherical pellets by balling. 


\subsection{Compaction}

A compaction is a form of pressure agglomeration in which drug particles or granules are forced together with or without formulation aids by a mechanical force to generate pellets of well defined shape and sizes. In compression, particles that are pretreated through dry blending or wet granulation followed by drying rearrange themselves to form a closely packed mass. At higher pressure, the particles are forced against each other and undergo elastic and plastic deformation. In extrusion spheronization, first the dry powder mix is agglomerated with the help of a binding liquid. Then it is processed in the extruder to produce high-density extrudates. These extrudates are finally converted to pellets on spheronizer. ${ }^{[8]}$

\subsubsection{Compression}

Compression is a pelletization process in which a mixture or blend of active ingredient and excipients are compacted under pressure to generate pellets of define shape and size. The pellets are small enough to be filled into capsule. ${ }^{[13]}$

\subsubsection{Extrusion / Spheronisation}

Extrusion / spheronisation is a multistage process for obtaining pellets with uniform size from wet granulates (extrudates).

The method involves the following main steps:

- The dry mixing of the ingredients, in order to achieve homogenous powder dispersions

- Wet massing, in which the powders are wet mixed to form a sufficiently plastic mass.

- An extrusion stage, in which the wet mass is shaped into cylindrical segments with a uniform diameter;

- The spheronisation stage, in which the small cylinders are rolled into solid spheres (spheroids);

- The drying of the spheroids, in order to achieve the desired final moisture content;

- Screening (optional), to achieve the desired narrow size distribution.

\subsubsection{Extrusion}

Extrusion consists in applying pressure to a wet mass until it passes through the calibrated openings of a screen or die plate of the extruder and further shaped into small extrudate segments.

As the mass passes through the extruder screen, the resulting extrudates eventually break under their own weight. Usually the extrudates have the same length. The extrudates must have enough plasticity in order to deform, but an excessive plasticity may lead to extrudates which stick to each other as they are collected and further processed in the spheroniser. The diameter of the segments and the final size of the spheroids depend on the diameter of the openings in the extruder screen. In order to obtain reproductible results, it is recommended to monitor extrusion parameters such as: feed rate, powder consumption, die temperature and compression chamber pressure.

\subsubsection{Spheronisation}

Spheronisation reffer to the formation of spherical particles from the small rods produced by extrusion. The essential part of the spheronizer is the friction plate. The indentation pattern on the plate can have various designs, which correspond to specific pur poses. The most common design is the cross hatch pattern with grooves intersecting each other at $90^{\circ}$ angles. In order to form spheroids, the extrudates are brought onto the rotating friction plate of the spheronizer, which imparts a rolling motion to the material $[10]$
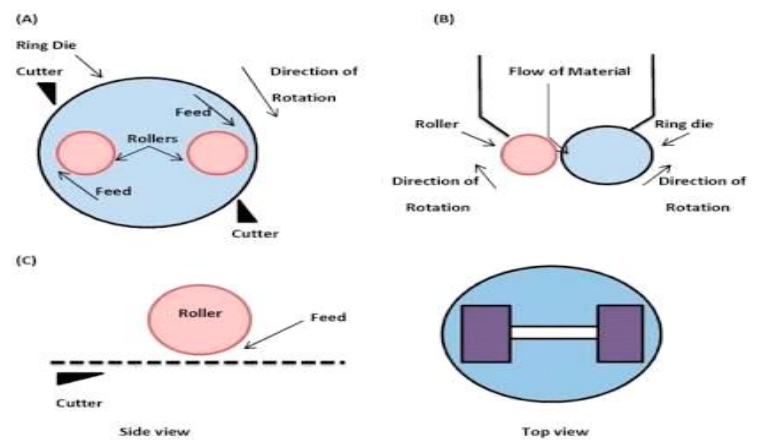

Figure 7. Extrusion spheronization. 


\section{A] Internal rollerB] roller external to die $\mathrm{C}]$ roller on flat die plate.}

\subsection{Drug layering}

It is the technique of pelletization which is used to coat or layer the seed material in powder, solution or suspension form. Initially, drug solution or suspension is prepared by using suitable solvent or non-solvent, respectively. Prepared solution or suspension of the drug is then sprayed over the inert spherical core, usually prepared by using microcrystalline cellulose or sugar. This process results in the formulation of varying sized pellets with inner core and outer shell having different composition. This technique has different types including: dry powder layering, solution and suspension layering and direct pelletization. ${ }^{[14]}$

\subsubsection{Powder layering}

This is the process in which consecutive layers of dry powder of the drug or/and excipients are deposited on preformed core. First, a binding solution is prepared having a suitable binder. The prepared binding solution is sprayed over the inert core of microcrystalline cellulose or sugar to prepare a sticky core with the ability to bind the drug powder over it. The drug, which is to be layered over prepared core, is grinded or micronized, if required, to prepared fine powder and then the finally divided powder is sprinkled over the inert spherical core in controlled manner to achieve uniform sized circular pallets Conventional coating pan is usually used for this purpose but it has few drawbacks like poor mixing and poor drying. The substrate particles are fluidized and suspended by heated and conditioned air. One or several nozzles atomize and spray the drug powder onto the substrate.

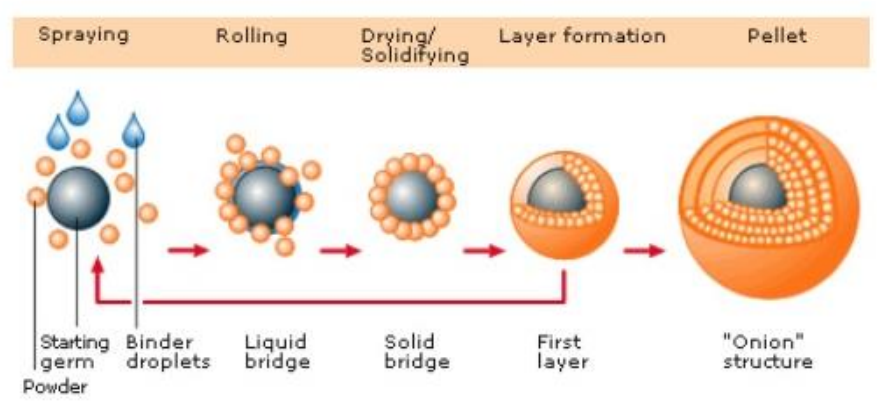

10.3.2 Suspension / Solution layering technique

This technique involves the deposition of successive layer of solution and /or suspension of drug substances and binders on starter seeds which may be inert material or crystal of granules of the same drug. In this technique drug particles and others component are dissolved or suspended in the application medium .The droplets impringe on the starter seeds or cores and spread evenly as the solution or suspension is sprayed on the cores. Followed by drying phase allows dissolved material to crystallize and form solid bridges between the cores and initial layer of the drug substances and among the successive layer of drug substances or polymer. Continue this process until the desired layer of drug or polymer formed. Consequently conventional coating press, fluidized bed centrifugal granulator of Wurster coater has been used successfully to manufacture pellets. The most common configuration for bottom spray coating is known as the Wurster system. In this study solution/ layering of neutral pellets has been conducted applying novel fluidized bed technology from .This technology claims to improve the product movement in defined direction in all the equipment by the Disk jet gas distribution plate. Further more, a 3 component spray nozzle is used in order to improve the film formation on the pellets due to constant and reproducible drop size distribution. Accessibility of clogged nozzles without stopping and interrupting the process makes the equipment advantageous in respect to Wurster system. Hüettlin's three 
component nozzle is an air nozzle with an additional channel through which a second gas or component can be introduced to create a special microclimate around the nozzle which prevents excessive spray drying or clogging of the nozzle. Such microclimates near nozzle apertures are very useful when a film former with a relatively high minimum film-forming temperature (MFT) issued [12].

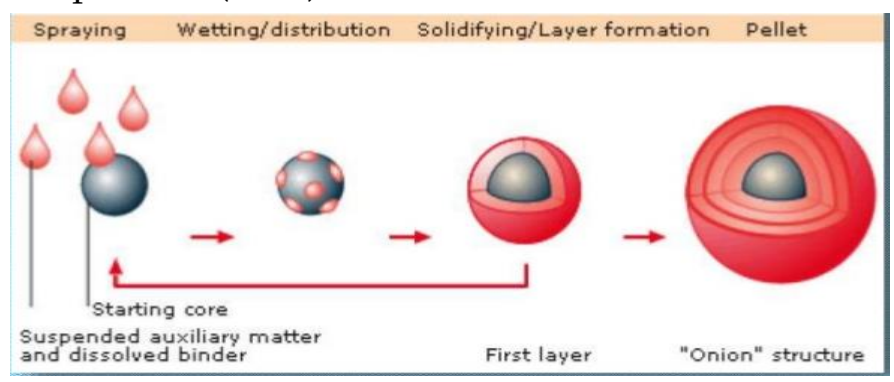

Figure 9. Principles of Solution and Suspension Layering Process

\subsection{Globulation}

Globulation or droplet formations consists two related processes spray drying and spray congealing.

\subsubsection{Spray drying:}

It is the process in which drugs in the suspension or solution without excipients are sprayed in to a hot stream to produce dry and more spherical particles. This process is commonly used for improving the dissolution rates; hence bioavailability of poorly soluble drugs.

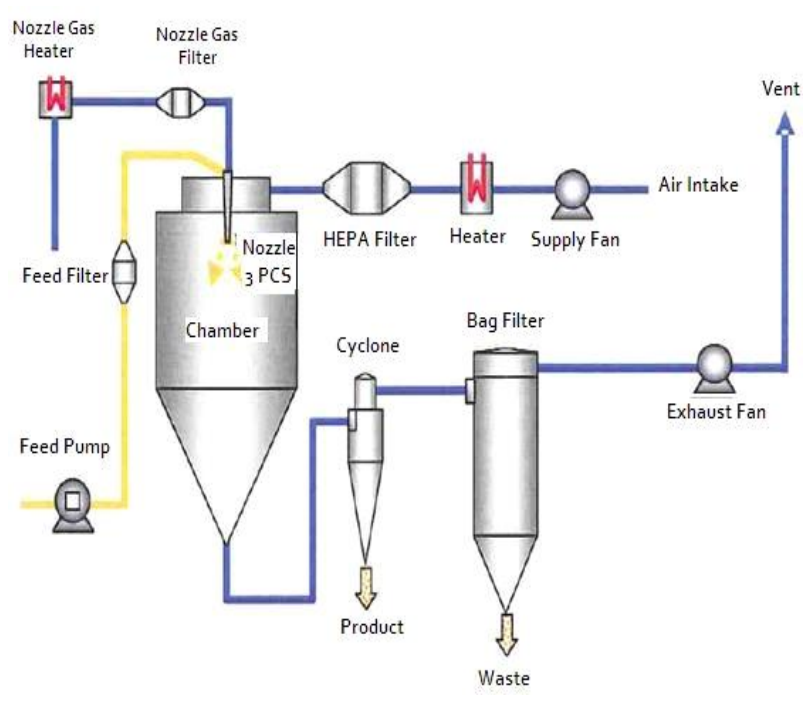

Figure 10. Spray drying.

\subsubsection{Spray congealing}

It is the process in which a drug is allowed to melt, disperse or dissolve in hot melts of gums, waxes or fatty acids, and is sprayed into an air chamber where the temperature is kept below the melting point of the formulation components, to produce spherical congealed pellets. Both immediate and controlled release pellets can be prepared in this process depending on the physiochemical properties of the ingredients and formulation variables ${ }^{[5]}$

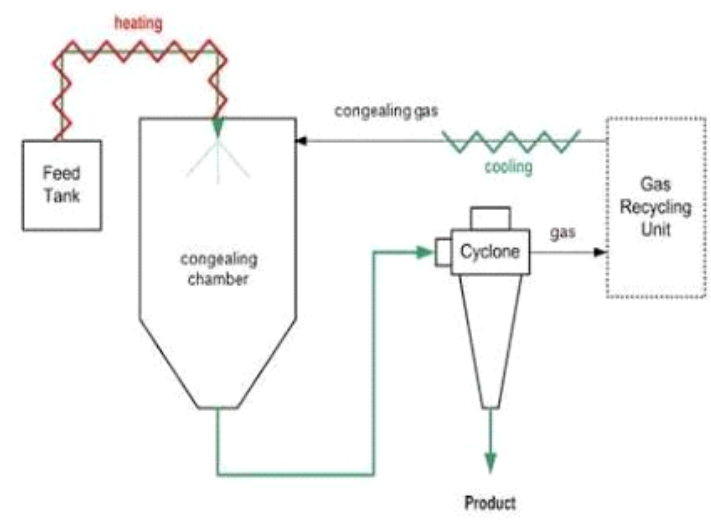

Figure 11. Spray congealing

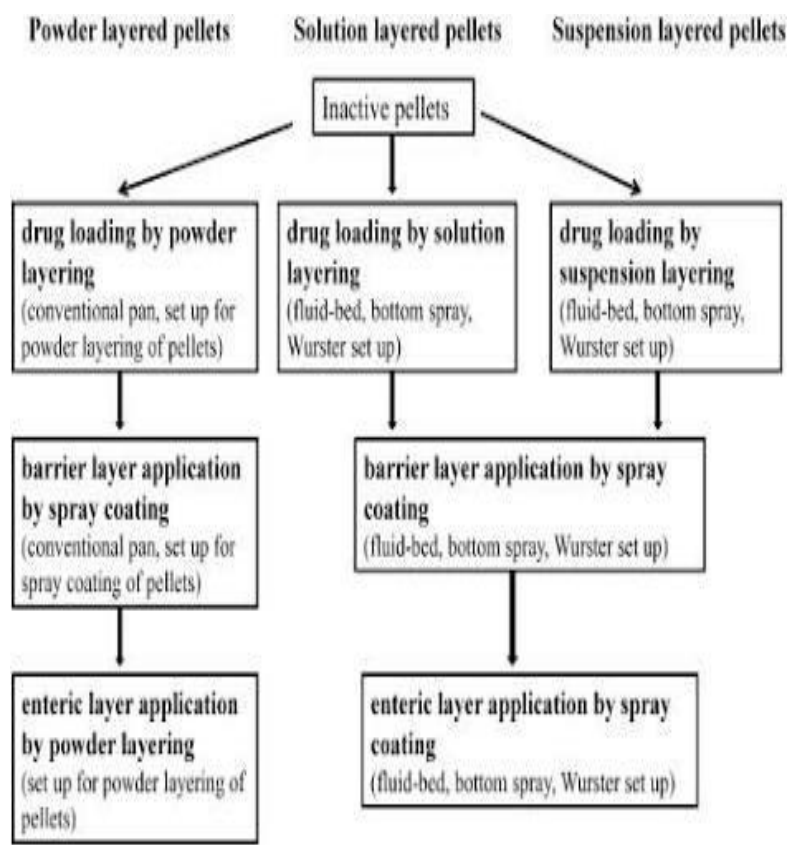

Figure 12. Representation of Powder layering, Solution layering and Suspension layering. 


\section{Factors affecting pelletization technique}

\section{Moisture content:}

Moisture in the wet mass brings cohesiveness to powder so that the wet mass can be extracted and spheronizer to give spherical shape. High moisture contents lead to agglomeration of pellets during the process of spheronization

\section{Rheological characteristics:}

The optimum rheological condition leads to good flow ability in order to extrudate the wet mass. The rheological variations make improper and nonuniform extrudate.

\section{Solubility of excipients and drug in granulating} fluid:

Soluble drug get dissolve in a granulating liquid. Thus increasing the volume of liquid phase leads to over wetting of pellets. But increase in wetting liquid increases plasticity but includes sticky mass

\section{Composition of granulating fluid:}

Besides water, alcohol, water/alcohol mixture, ethyl ether, dilute acetic acid, isopropyl alcohol is used as a granulating liquid. Aqueous polymer dispersion containing HPMC, PVP, etc can also be used as granulating fluid.

\section{Physical properties of starting material:}

Quality of pellets depend not only composition but also on different grades of the same product. The swelling property of material used in pelletization technique decides the release rate of drug in pellets.

\section{Speed of Spheronizer:}

It affects the size, hardness, sphericity and density of pellets. The high speed gives high sphericity, lower friability, smooth surface and higher crushing strength.

\section{Extrusion screen:}

The quality of pellets is greatly influenced by the characteristics of orifice of the screen. And increase in orifice dimension resulted in increased mean pellet size. The increase in orifice depth decreased with the presence of water at the extrudate surface. ${ }^{[1]}$

\section{APPLICATIONS OF PELLETS}

\subsection{Taste masking}

Micro pellets are ideal for products where perfect abatement of taste is required. Although various technique have been utilized to mask the bitter taste of a drug such as the addition of sweeteners and flavors, filling in capsules, coating with water insoluble polymers or $\mathrm{pH}$ dependent soluble polymers, complexing with ion-exchange resins, microencapsulation with various polymers, complexing with cyclodextrin and chemical modifications such as the use of insoluble prodrugs, few reports have described the masking of unpleasant taste without lowering of bioavailability especially for oral products. The micropelletization technique solves difficult taste masking problems while maintaining a high degree of bioavailability due to their high surface area, especially for oral products. Furthermore, because of the special design of the manufacturing process, dust fractions that representing an uncoated fragments which could cause taste problems are absent in micro pellets. Many products, such as antibiotics (clarithromycin, roxithromycin and cephelexin) and antiinflammatory drugs with a prohibitively bitter taste, can now be formulated in products with high patient compliance, thus markedly increasing the sales potential of the product.

\subsection{Immediate release}

Administering drugs in pellet form leads to an increased surface area as compared to traditional compressed tablets and capsules. This would considerably reduce the time required for disintegration and have the potential for use in rapidly dispersible tablet.

\subsection{Sustained release}

The pellet form provides a smoother absorption 
profile from the gastrointestinal tract as the beads pass gradually through the stomach in to the small intestine at a steady rate. Pellets are being increasingly used in the manufacture of sustained release dosage form of drugs. The advantage of the dosage form is well known and some examples are given below:

- Extend day time and night time activity of the drugs,

- Potential for reduced incidence of side effects,

- Reduced dosage frequency of dosage forms,

- Increased patient compliance, patients who are required to take 2 or more doses of formulation a day are thought to be less likely to forget a dose then if they are required to take 3 or 4 times a day,

- Potential lower daily cost to patient due to fewer dosage units.

\subsection{Chemically incompatible products}

At times such ingredients are required to be delivered in a single dose. In the compressed tablet dosage form separate tablets would have to be administered, but the pellets can be administered in a single capsule.

\subsection{Varying dosage without reformulation}

Pellets have excellent flow properties, due to this; they can be conveniently used for Filling capsules and the manufacturer can vary the dosage by varying the capsule size without reformulating the product. [12]

\section{Conclusion}

Development of pelletization has acquired the market of novel drug delivery involving both the controlled as well as immediate release. They are having simple design, high efficiency of producing spherical pellets, flexibility and robustness. Pelletization lays the scope for different oral immediate or controlled delivery system. Due to its simple design, greater flexibility, efficiency of producing spherical pellets and fast processing; it has found a special place in the Pharmaceutical industry. Pellets are the multi-unit dosage forms which offer improved safety and efficacy of the active ingredients with excellent flow properties which is then fabricated in single dosage form.

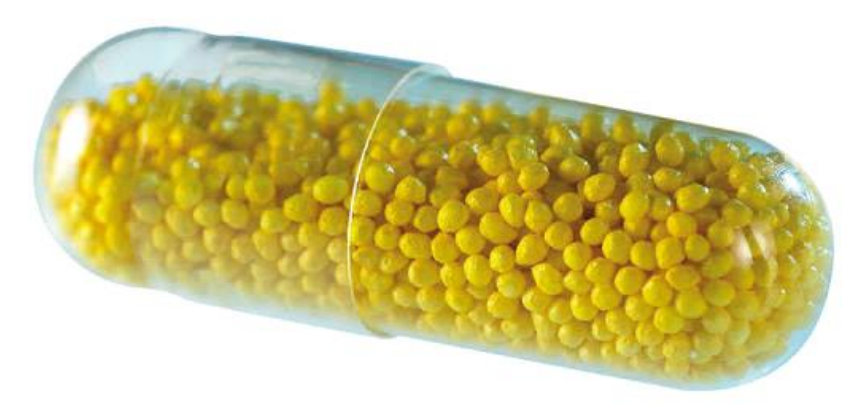

\section{CONCLUSION}

Pellets are spherical or nearly spherical, free-flowing granules with a narrow size distribution, typically varying between 500 and $1500 \mu \mathrm{m}$ for pharmaceutical applications. They are generally produced via a pelletization process whereby a powder blend consisting of an API and excipient particles is agglomerated into spherical granules. This review article deals with various aspects of the extrusionspheronization technique. Pelletization is a technique to convert drugs or excipients to small free flowing, spherical or semi spherical units, which are produced by agglomerating fine powdered drugs/ excipients with a binder solution. Pellets range in size, typically, between $0.5-2 \mathrm{~mm}$. In relation to pharmaceuticals, pellets offer high degree of flexibility in design and development of oral dosage form. Pelletization technique help in the formation of spherical beads or pellets having a diameter $0.5-1.5 \mathrm{~mm}$ which can be eventually coated for preparation of modified release dosage form. The manufacturing techniques include Drug layering, Extrusion-Spheronization, Cryopelletization, Compression, Balling, Hot-Melt Extrusion Technology, Freeze pelletization, Spraydrying \& Spray-congealing. Factors affecting pelletization technique and advantages, disadvantages of pellets are discussed. 


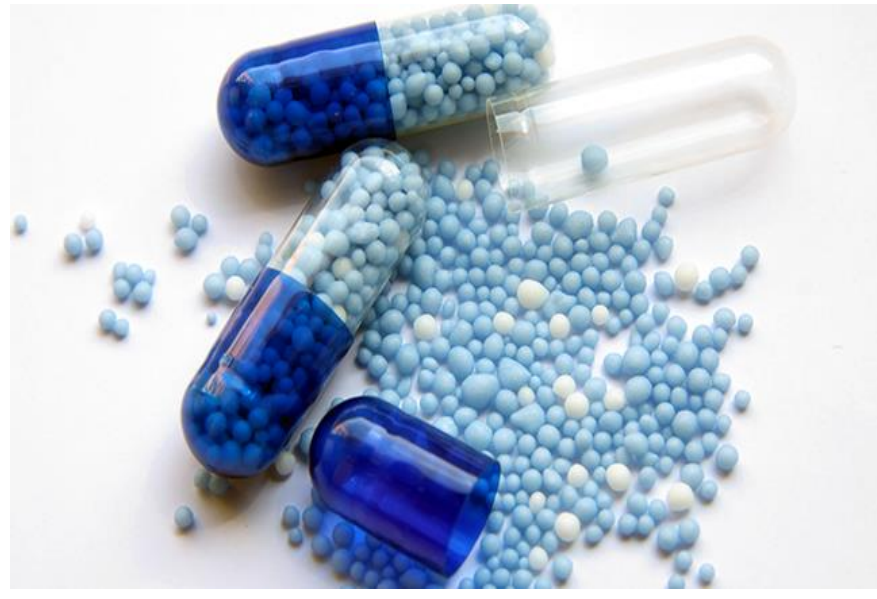

Figure 13. Pellets to preaparing capsule pelletization technique.

\section{REFERENCES}

[1]. Slideshare.net

[2]. Review Extrusion- spheronization a promising pelletization technique: In-depth review. Asian journal of pharmaceutical sciences 11 (2016) 684-699.

[3]. International Journal of Pharmaceutical Development \& Technology. Pelletization Technique in drug delivery system- a review. Veena mc. et al. / IJPDT / 3 (1), 2013, 13-22.

[4]. Slide Player.Pellets: as Pharmaceutical Dosage Form . Journal of Advanced Drug Delivery (JADD).Volume 3, Issue 3 May- June -2016.

[5]. Multiparticular Drug Delivery System: Pelletization. Received 3 December 2011, Accepted 10 December 2011.

[6]. Pelletization Technology: Methods and Applications -A Review. Research J. Pharm. and Tech.8 (2): February 2015.

[7]. Multi unit drug delivery system - A brief review of pelletization technique. Anwar, et al. Int J Pharm 2014; 4(3): 234-237

[8]. International Research Journal Of Pharmacy.Pellets And Pelletization Techniques:A Critical Review. Deb Ratul et al.int.res.J.Pharm.2013,4(4).
[9]. Pelletization Techniques Used In Pharmaceutical Fields. Practica Farmaceutică Vol. 4, Nr. 3-4, An 201

[10]. Pelletization Techniques: A Review. Journal Of Pharmaceutical Science And Bioscientific Research (JPSBR). JPSBR: Volume 5, Issue 3: 2015 (244-248).

[11]. Recent Advances In Pellets And Pelletization Techniques For Oral Sustained Release Drug Delivery. World Journal of Pharmaceutical Research. Vol 4, Issue 3, 2015.

[12]. Multiple Unit Dosage Form pellets And Pelletization Techniques: An Overview .Kumar Vikash et al/IJRAP 2011, 2 (1)121-125

[13]. Pellets Ans Pelletization: Emerging Trends In The Pharma Indusry. Acta Poloniae Pharmaceutica ñ Drug Research, Vol. 73 No. 6 pp. 1415ñ1425, 2016. usion) a conclusion is the last part of something, its end or result.

\section{Cite this article as :}

Harsha V Sonaye, Mohmad Rafik Y. Shaikh, Rubina Shaikh, "Pelletization Techniques. The Pharma Review", International Journal of Scientific Research in Science and Technology (IJSRST), Online ISSN : 2395-602X, Print ISSN : 2395-6011, Volume 6 Issue 2, pp. 690-700, March-April 2019. Available at doi : https://doi.org/10.32628/IJSRST1962147 Journal URL : http://ijsrst.com/IJSRST1962147 